\title{
Cortical and subcortical neuroanatomical signatures of schizotypy in 3004 individuals assessed in a worldwide ENIGMA study
}

\begin{abstract}
Matthias Kirschner (iD ${ }^{1,2,38}$, Benazir Hodzic-Santor ${ }^{1,38}$, Mathilde Antoniades ${ }^{3}$, Igor Nenadic ${ }^{4}$, Tilo Kircher ${ }^{4}$, Axel Krug ${ }^{4,5}$, Tina Meller ${ }^{4}$, Dominik Grotegerd $^{6}$, Alex Fornito (iD ${ }^{7}$, Aurina Arnatkeviciute ${ }^{7}$, Mark A. Bellgrove (D) ${ }^{7}$, Jeggan Tiego (iD) ${ }^{7}$, Udo Dannlowski (iD ${ }^{6}$, Katharina Koch ${ }^{6}$, Carina Hülsmann ${ }^{6}$, Harald Kugel $\mathbb{D}^{8}{ }^{8}$, Verena Enneking ${ }^{6}$, Melissa Klug ${ }^{6}$, Elisabeth J. Leehr ${ }^{6}$, Joscha Böhnlein (iD ${ }^{6}$, Marius Gruber ${ }^{6}$, David Mehler ${ }^{6}$, Pamela DeRosse (iD ${ }^{9,10,11}$, Ashley Moyett ${ }^{9}$, Bernhard T. Baune ${ }^{6,12}$, Melissa Green ${ }^{13,14}$, Yann Quidé ${ }^{13,14}$, Christos Pantelis (iD ${ }^{15}$, Raymond Chan (iD ${ }^{16}$, Yi Wang ${ }^{16}$, Ulrich Ettinger ${ }^{17}$, Martin Debbané ${ }^{18}$, Melodie Derome ${ }^{18}$, Christian Gaser ${ }^{19}$, Bianca Besteher ${ }^{19}$, Kelly Diederen ${ }^{3}$, Tom J. Spencer ${ }^{3}$, Paul Fletcher ${ }^{20}$, Wulf Rössler ${ }^{21,22,23}$, Lukasz Smigielski ${ }^{21}$, Veena Kumari (iD ${ }^{24}$, Preethi Premkumar ${ }^{24}$, Haeme R. P. Park ${ }^{25}$, Kristina Wiebels (iD ${ }^{25}$, Imke Lemmers-Jansen ${ }^{26}$, James Gilleen ${ }^{3,27}$, Paul Allen ${ }^{27}$, Petya Kozhuharova ${ }^{27}$, Jan-Bernard Marsman ${ }^{28}$, Irina Lebedeva ${ }^{29}$, Alexander Tomyshev ${ }^{29}$, Anna Mukhorina (iD) ${ }^{29}$, Stefan Kaiser ${ }^{30}$, Anne-Kathrin Fett ${ }^{3,31}$, Iris Sommer ${ }^{28}$, Sanne Schuite-Koops ${ }^{28}$, Casey Paquola ${ }^{1}$, Sara Larivière (D) ${ }^{1}$, Boris Bernhardt ${ }^{1}$, Alain Dagher (iD ${ }^{1}$, Phillip Grant ${ }^{32}$, Theo G. M. van Erp $\mathbb{D}^{33,34}$, Jessica A. Turner $\mathbb{D}^{35}$, Paul M. Thompson ${ }^{36}$, André Aleman ${ }^{28}$ and Gemma Modinos $\mathbb{D}^{3,37 凶}$
\end{abstract}

(c) The Author(s) 2021

Neuroanatomical abnormalities have been reported along a continuum from at-risk stages, including high schizotypy, to early and chronic psychosis. However, a comprehensive neuroanatomical mapping of schizotypy remains to be established. The authors conducted the first large-scale meta-analyses of cortical and subcortical morphometric patterns of schizotypy in healthy individuals, and compared these patterns with neuroanatomical abnormalities observed in major psychiatric disorders. The sample comprised 3004 unmedicated healthy individuals (12-68 years, 46.5\% male) from 29 cohorts of the worldwide ENIGMA Schizotypy working group. Cortical and subcortical effect size maps with schizotypy scores were generated using standardized methods. Pattern similarities were assessed between the schizotypy-related cortical and subcortical maps and effect size maps from comparisons of schizophrenia (SZ), bipolar disorder (BD) and major depression (MDD) patients with controls. Thicker right medial orbitofrontal/ ventromedial prefrontal cortex (mOFC/vmPFC) was associated with higher schizotypy scores $\left(r=0.067, p_{\mathrm{FDR}}=0.02\right)$. The cortical thickness profile in schizotypy was positively correlated with cortical abnormalities in SZ $\left(r=0.285, p_{\text {spin }}=0.024\right)$, but not BD $(r=$ $\left.0.166, p_{\text {spin }}=0.205\right)$ or MDD $\left(r=-0.274, p_{\text {spin }}=0.073\right)$. The schizotypy-related subcortical volume pattern was negatively correlated with subcortical abnormalities in SZ ( $\left.\mathrm{rho}=-0.690, p_{\text {spin }}=0.006\right), \mathrm{BD}$ ( $\mathrm{rho}=-0.672, p_{\text {spin }}=0.009$ ), and $\mathrm{MDD}$ ( $\mathrm{rho}=$ $\left.-0.692, p_{\text {spin }}=0.004\right)$. Comprehensive mapping of schizotypy-related brain morphometry in the general population revealed a significant relationship between higher schizotypy and thicker mOFC/vmPFC, in the absence of confounding effects due to antipsychotic medication or disease chronicity. The cortical pattern similarity between schizotypy and schizophrenia yields new insights into a dimensional neurobiological continuity across the extended psychosis phenotype.

Molecular Psychiatry (2022) 27:1167-1176; https://doi.org/10.1038/s41380-021-01359-9

\section{INTRODUCTION}

Schizophrenia (SZ) and related psychotic disorders are increasingly recognized as clinical manifestations of quantitative characteristics that are continuously distributed in the general population. In this regard, schizotypy has been linked to trait-like vulnerabilities along a continuum from health to (psychotic) illness [1-3]. Since the early descriptions from Rado and Meehl [4-6], different continuous models of schizotypy and its relationship to SZ have been proposed [7]. A fully-dimensional model assumes that schizotypal traits are not inherently associated with illness but sit squarely within the realm of health, and that clinical manifestations (such as SZ and related psychoses) result from multifactorial breakdown-processes within some (but not all) highly schizotypal individuals in the general population [7-9].

Schizotypy is multi-faceted in nature [10-12], with clinical conditions thought to reflect qualitatively different schizotypy dimensions which interact with general psychopathology [13]. Specifically, schizotypy dimensions closely resemble those of SZ and reflect distinct patterns of positive, negative, and disorganized symptoms [12, 14]. Psychometric measures of schizotypy have been used to assess individuals at clinical-high risk (CHR) for psychosis, and were predictive of conversion to psychosis in both CHR samples and in the general population [1, 15]. Moreover, individuals with high schizotypy exhibit genetic, neurobiological,

A full list of author affiliations appears at the end of the paper. 
cognitive, and behavioral characteristics similar (albeit attenuated) to patients with SZ spectrum disorders [16-23]. Thus, in a neurodevelopmental and multifactorial view of psychosis [24, 25], high schizotypy represents a liability that in concert with other environmental and biological risk factors increases the risk for developing psychosis $[9,26]$. Of note, this is not contradictory to a fully-dimensional model of schizotypy conceptualizing schizotypal traits as both healthy variations as well as predisposing to psychosis [16]. From a psychosis continuum perspective, healthy individuals with high schizotypy could therefore be placed on the left side, followed by CHR individuals, with SZ spectrum disorders toward the right-most end of the continuum [1].

Converging evidence from neuroimaging research also supports a dimensional view of psychosis. Cortical neuroanatomical abnormalities have been reported in individuals with treatment resistant SZ [27], chronic SZ [28], first-episode psychosis [29], CHR $[30,31]$, schizotypal personality disorders [32], and individuals with non-clinical psychotic symptoms [33]. Recently, the SZ Working Group within the ENIGMA (Enhancing Neuro Imaging Genetics through Meta Analysis) consortium provided meta-analytic evidence for robust abnormalities in subcortical volumes [34], cortical thickness (CT), and surface area (SA) in SZ [28], while also indicating that these abnormalities may be influenced by illness severity and antipsychotic medication [28, 34].

In this context, the study of schizotypy offers a unique opportunity to identify neuroanatomical signatures related to psychosis vulnerability without the common confounds of antipsychotic treatment or disease chronicity [32]. Over the last decade, there has been increased focus on the neuroanatomy of schizotypy [19, 35-43]. The majority of these single studies examined morphometric brain correlates related to total schizotypy scores (including all schizotypy dimensions) [33, 36, 39, 4345]. Others applied combined approaches using both total scores and schizotypy dimensions [35, 46], or multivariate statistics [42], and reported evidence for shared cross-dimensional neuroanatomical abnormalities of total schizotypy as well as patterns related to distinct dimensions $[23,35,41,42]$. However, while these studies consistently reported morphometric abnormalities associated with high levels of schizotypy, most studies included relatively small samples and the directionality of the findings was largely inconclusive (e.g., larger or smaller thickness/volumes). Furthermore, almost all studies focused on CT or cortical gray matter volume, rendering the schizotypy literature on subcortical volume and SA relatively scarce.

To address these issues, the Schizotypy Working Group within the ENIGMA consortium brought together schizotypy researchers worldwide toward the first large-scale meta-analysis of regional $\mathrm{CT}$, SA and subcortical volumes in schizotypy. We report the first comprehensive neuroanatomical mapping of overall schizotypy using standardized methods in 29 datasets worldwide. To provide meta-analytical evidence that reflects the large body of single neuroanatomical studies in schizotypy presented above, we focused on total schizotypy scores. In line with a dimensional view of psychosis, our main models capitalized on partial correlation effect sizes with continuous measures of schizotypy. Based on recent work in SZ $[28,47,48]$, we hypothesized stronger effect sizes for schizotypy-related CT effects, compared to SA and subcortical volumes. Our second aim was to examine the shared morphometric characteristics of schizotypy with previously reported structural abnormalities in SZ, bipolar disorder (BD) and major depression. To this end, we correlated subcortical and cortical effect size maps derived from the present meta-analysis with recently published effect size maps from the ENIGMA consortia of these three major psychiatric disorders. We hypothesized that schizotypy-related morphometric patterns would be most similar to morphometric patterns of $S Z$, relative to $B D$ and major depression.

\section{METHODS}

\section{Study sample}

Cortical thickness and surface area. Twenty-nine cross-sectional study samples totaling 3004 unmedicated healthy individuals with varying levels of schizotypy (below) passed Quality Control (QC) and contributed to the cortical meta-analysis. Sample-size average of mean (range) age across samples for this meta-analysis was 30.1 (12-68) years and samples were on average $46.5 \%$ male (27-100\%) (Table S1 and Fig. S1).

Subcortical volumes. From the same 29 cross-sectional study samples, data from 2990 healthy, unmedicated individuals passed QC and contributed to the subcortical meta-analysis. Sample-size average of mean (range) age across samples was 30.1 (12.1-67.8) years and samples were on average $46.5 \%$ male (26.6-100\%) (Table S2 and Fig. S1).

All participants included in this meta-analysis had no current or past history of any psychiatric or neurological disorder, and had no current or past history of antipsychotic treatment. Each study sample was collected with participants' written informed consent approved by local institutional review boards.

\section{Assessment of schizotypy}

Across all 29 centers, schizotypy was assessed with well-validated instruments, including the Chapman scales [49-51], the Community Assessment of Psychotic Experiences (CAPE) [52], the Schizotypal Personality Questionnaire (SPQ) [53], the Oxford-Liverpool Inventory of Feelings and Experiences (O-LIFE) [54], and the Rust Inventory of Schizotypal Cognitions (RISC) [55] (for details of schizotypy measures see Table S3). Overall, 18 sites used the SPQ or brief version (SPQ-B), six sites used the CAPE, three sites used the O-LIFE, one site used the Chapman scale, and one site used the RISC. Because our hypotheses involved the association between neuroanatomy with overall schizotypal traits, only total schizotypy scores were included. Associations based on different schizotypy dimensions are to be reported elsewhere [56].

\section{Image acquisition and processing}

Following published ENIGMA pipelines [28, 34], all sites processed T1weighted structural scans using FreeSurfer $[57,58]$ (http://surfer.nmr.mgh. harvard.edu) and extracted CT and SA for 70 Desikan-Killiany (DK) atlas regions [59] (34 regions per hemisphere; 1 left and right hemisphere mean thickness or total SA) (Table S4). Simultaneously, subcortical volumes of 16 brain structures including left and right lateral ventricle, thalamus, caudate, putamen, pallidum, accumbens, hippocampus and amygdala, and intracranial volume (ICV) were extracted (Table S5). Number of scanners, vendor, strength, sequence, acquisition parameters, and FreeSurfer versions are provided in Table S6. QC followed standard ENIGMA protocols at each site before analysis. For subcortical data, all regions of interest (ROIs) with a volume deviating from the mean by more than 1.5 times from the interquartile range were identified and only included after additional visual inspection. For cortical data, ENIGMA's quality assurance protocol was performed (http://enigma.usc.edu/protocols/imaging-protocols) including visual inspection of the cortical segmentation and region-byregion removal of values from incorrect segmentations.

\section{Statistical meta-analyses}

Cortical measures. Continuous models were fitted to examine the relationship between schizotypy and CT (or SA) in each sample. Partial correlation analysis (pcor.test, $\mathrm{R}$ version 3.6.0, R Foundation for Statistical Computing, Vienna, Austria) was used to assess the association between the CT (or SA) of left and right DK atlas regions with total schizotypy scores including age, sex, and global mean CT (or total SA) as covariates (continuous model 1) and secondarily excluding global mean CT (or total SA) (continuous model 2, Tables S7 and S8). For multi-scanner studies $(n=3)$, binary dummy covariates ( $\mathrm{n}-1$ scanners) were included within each local site model prior to metaanalysis to account for potential differences that may emerge across different scanners, consistent with the standard meta-analysis approach by the ENIGMA project [28, 34, 65-68]. To account for potential confounding effects of smoking on brain morphometry [60,61], we were able to use data on smoking status that was available for a subsample of participants $(n=1303)$. Within this subsample, the continuous model 1 including age, sex and global mean $\mathrm{CT}$ was repeated with and without smoking as additional covariate to assess whether smoking status would affect the relationship between schizotypy and CT (Tables S9 and S10). 
Subcortical volumes. Similar to the cortical analyses, continuous models were applied to examine the relationship between schizotypy scores and subcortical volume for each ROI in each sample. To this end, partial correlation analysis was used to test correlations between the left and right subcortical volumes with total schizotypy scores. The main model included age, sex, and ICV as covariates. For multi-scanner studies $(n=3)$, binary dummy covariates were included to account for differences that may emerge across scanners, as described above. As for the cortical analysis, partial correlation analysis was repeated in a subsample of $n=1303$ participants including smoking status as covariate and are reported in Tables S11 and S12.

Meta-analyses. For cortical and subcortical measures, Pearson's $r$ effect sizes from the partial correlations using schizotypy scores as a continuous predictor were meta-analyzed in separate random effects models to account for between study differences ( $r$ ma function, metafor package for $\mathrm{R}$ 3.6.0) [62]. The false discovery rate $(\mathrm{FDR})$ procedure $\left(p_{\mathrm{FDR}}<0.05\right)$ was used to control for multiple comparisons $[63,64]$. Meta-analyses were adjusted for sample sizes across different sites and results were weighted for sample sizes. Possible confounding effects of schizotypy questionnaire type, FreeSurfer version, number of scanners, and scanner field strength were examined using moderator analyses (Tables S13-S16).

Cortical and subcortical pattern similarity between schizotypy and major psychiatric disorders. To answer our second question on how overall CT and subcortical volumes in schizotypy relate to the neuroanatomical patterns observed in SZ, BD and major depression, we correlated the schizotypy-related effect size maps of correlation coefficient $r$ for CT and SA (age, sex corrected), as well as for subcortical volumes (age, sex, ICV corrected) with the Cohen's d maps derived from recently published metaanalyses of case-control studies by the ENIGMA SZ $[28,34], \operatorname{BD}[65,66]$, and depression (MDD) working groups $[67,68]$. This approach followed previous studies correlating cortical effects size maps between different disorders $[69,70]$ or disorders and regional network features of the brain [71]. Specifically, we applied a recently developed approach from the ENIGMA Epilepsy Working Group using Pearson correlation to investigate spatial pattern similarity of cortical effect size maps [71]. Statistical significance of all cortical pattern correlations was assessed using spin permutation tests correcting for spatial autocorrelation [72, 73], following recent work from the ENIGMA Epilepsy Group [71]. In this framework, null models are generated by projecting the spatial coordinates of cortical data onto the surface spheres, applying randomly sampled rotations $(10,000$ repetitions), and reassigning cortical data (here effect size values) [72]. The original correlation coefficients are then compared against the empirical distribution of spatially permuted correlation coefficients. This spatial autocorrelation approach and the adequate control of false positives has been comprehensively validated for parcellated ROI data, including resolutions of 68 parcellations as in our study [73].

Spatial pattern similarity between subcortical maps was examined using Spearman rank correlations to account for potential outlier effects in small sample sizes [74]. Statistical significance testing followed a similar approach as the spin permutation from the cortical analysis with the exception that subcortical labels were randomly shuffled as opposed to being projected onto spheres [71]. We hypothesized that the schizotypyrelated cortical or subcortical effect size maps would be positively correlated with the corresponding SZ effect size maps, suggesting neuroanatomical continuity of the SZ spectrum. We additionally hypothesized that the strength of cortical and subcortical relationships would follow a psychosis-to-affective disorder axis ( $S Z>B D>M D D)$. To this end, pairwise comparisons of the correlations between schizotypy-SZ with schizotypy-BD and schizotypy-MDD were performed using one-tailed Steiger's test (cocor package, R 3.6.0) [75].

\section{RESULTS}

\section{Cortical thickness and schizotypy}

Meta-analysis of the continuous relationship between $C T$ and schizotypy $(n=3004)$ revealed positive correlations between higher schizotypy and greater thickness of the medial orbitofrontal cortex/ ventromedial prefrontal cortex (mOFC/vmPFC) (left: $r=0.057, p_{\text {unc }}$ $=0.004,95 \% \mathrm{Cl}[0.02-0.09]$; right: $r=0.067, p_{\text {unc }}<0.001,95 \% \mathrm{Cl}$ $[0.03,0.10])$ as well the frontal pole (left: $r=0.046, p_{\text {unc }}=0.021,95 \%$ Cl [0.01, 0.08]; right: $\left.r=0.050, p_{\text {unc }}=0.007,95 \% \mathrm{Cl}[0.01,0.09]\right)$ (Fig. 1A, Table S17). The positive association between greater right
mOFC/vmPFC thickness and higher schizotypy remained significant after FDR correction $\left(r=0.067, p_{\mathrm{FDR}}=0.02\right)$ (Fig. 2A/C). Moderator analyses did not reveal any significant effects of type of schizotypy questionnaire, FreeSurfer version, number of scanners or scanner field strength on the associations between $\mathrm{CT}$ and schizotypy (all $p_{\mathrm{FDR}}>0.05$ ) (Tables S10-S13).

\section{Surface area and schizotypy}

Higher schizotypy was associated with lower SA in the left parahippocampal gyrus $\left(r=-0.056, p_{\text {unc }}=0.006,95 \% \mathrm{Cl}[-0.096\right.$, $-0.016])$ and left fusiform cortex $\left(r=-0.043, p_{\text {unc }}=0.038,95 \% \mathrm{Cl}\right.$ $[-0.084,-0.002])$ (Table S18), but these effects did not survive correction for multiple comparisons $\left(p_{\mathrm{FDR}}<0.05\right)$ (Figs. 1B, 2B).

\section{Subcortical volume and schizotypy}

When examining the continuous relationship between subcortical volumes of 16 brain structures and schizotypy scores $(n=2990)$, only non-significant correlations were found, ranging from negative correlations between higher schizotypy and lower volume in the right pallidum $\left(r=-0.032, p_{\text {unc }}=0.084,95 \% \mathrm{Cl}\right.$ $[-0.07,0.004])$, to positive correlations between higher schizotypy and greater volume in the right accumbens $\left(r=0.020, p_{\text {unc }}=\right.$ $0.388,95 \% \mathrm{Cl}[-0.02,0.06])$, right amygdala $\left(r=0.019, p_{\text {unc }}=\right.$ $0.444,95 \% \mathrm{Cl}[-0.03,0.07])$, and right hippocampus $(r=0.022$, $\left.p_{\text {unc }}=0.229,95 \% \mathrm{Cl}[-0.01,0.06]\right)$ (Fig. 3). All subcortical results from the continuous analysis are summarized in Table S19.

\section{Cortical and subcortical pattern similarity between schizotypy and major psychiatric disorders}

Correlations of cortical maps revealed significant positive associations between increasing levels of schizotypy-related morphometry (partial correlation $r$ ) and Cohen's d maps from ENIGMA SZ ( $r=$ $\left.0.285, p_{\text {spin }}=0.024\right)$, but not ENIGMA BD $\left(r=0.166, p_{\text {spin }}=0.205\right)$ or ENIGMA MDD $\left(r=-0.274, p_{\text {spin }}=0.073\right) \quad$ (Fig. 4). Pairwise comparisons of these correlations revealed that the relationship between schizotypy and SZ cortical patterns was significantly stronger than the correlations between schizotypy and $\mathrm{MDD}(Z=$ $4.063, p$ value $<0.0001,95 \% \mathrm{Cl}[0.3-0.8])$ and at trend level to the correlation between schizotypy and $\mathrm{BD}(Z=1.462, p=0.072,95 \%$ $\mathrm{Cl}[-0.04-0.8])$. These findings indicate that the schizotypy-related CT pattern is more closely related to cortical brain alterations in SZ compared to BD and MDD.

Repeating the cortical pattern similarity analyses with SA effect size maps of schizotypy and all three psychiatric disorder maps revealed that the schizotypy related SA pattern was not correlated with the SA patterns of either SZ $\left(r=-0.009 p_{\text {spin }}=0.953\right), \mathrm{BP}(r$ $\left.=-0.221 p_{\text {spin }}=0.139\right)$ or MDD $\left(r=-0.05 p_{\text {spin }}=0.720\right)$. These findings suggest that the observed cortical pattern similarity between schizotypy and SZ is specific for CT rather than SA.

In terms of subcortical volumes, effect sizes for schizotypyrelated patterns (partial correlation $r$ ) were significantly negatively associated with the profile of Cohen's $d$ values for subcortical volume abnormalities in ENIGMA SZ (rho $=-0.690, p_{\text {spin }}=0.006$ ), ENIGMA BD (rho $=-0.672, p_{\text {spin }}=0.009$ ), and ENIGMA MDD (rho $=-0.692, \quad p_{\text {spin }}=0.004$ ) (Fig. 5). In other words, subcortical volumes in schizotypy showed an opposite pattern to subcortical abnormalities in SZ, BD or MDD, with similar magnitudes for all three correlations. The largest differences in effect sizes between subcortical profiles of schizotypy and all three psychiatric disorders were observed in the hippocampus. In particular, patients with SZ but also BD and MDD showed smaller hippocampal volume compared to controls, while in healthy individuals higher schizotypy was (weakly) positively correlated with greater hippocampus volume (Fig. 5). In addition, for SZ the opposite effect was observed for pallidum volume, displaying larger volume in patients compared to controls, and a (weak) negative correlation between smaller volume and higher schizotypy in healthy individuals (Fig. 5). 


\section{A| Cortical thickness}

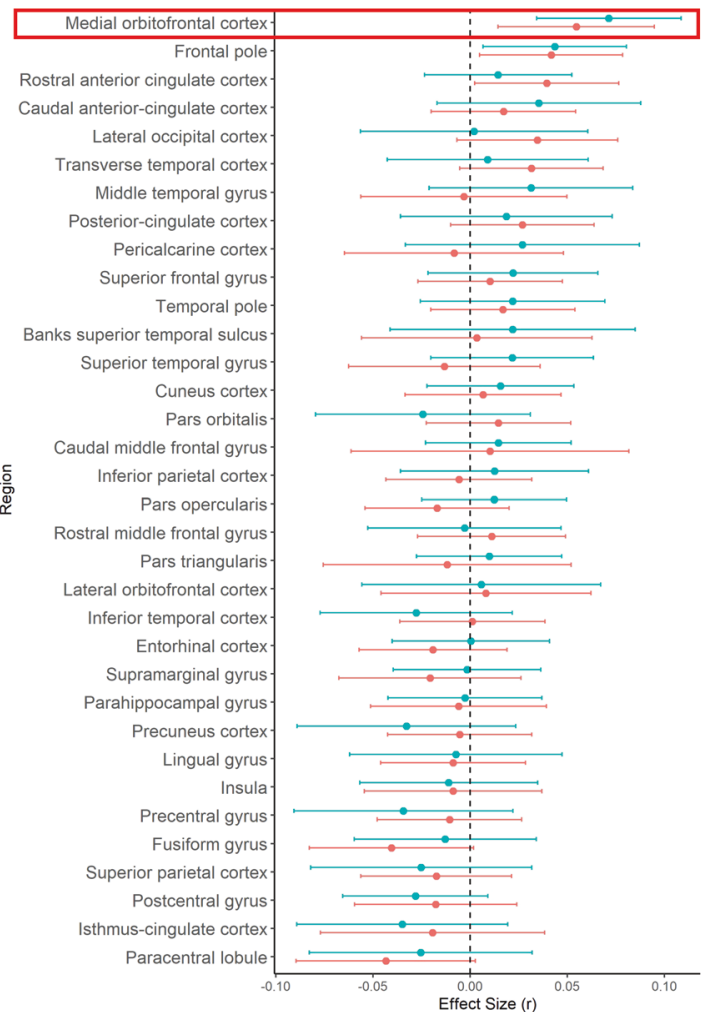

B| Surface area

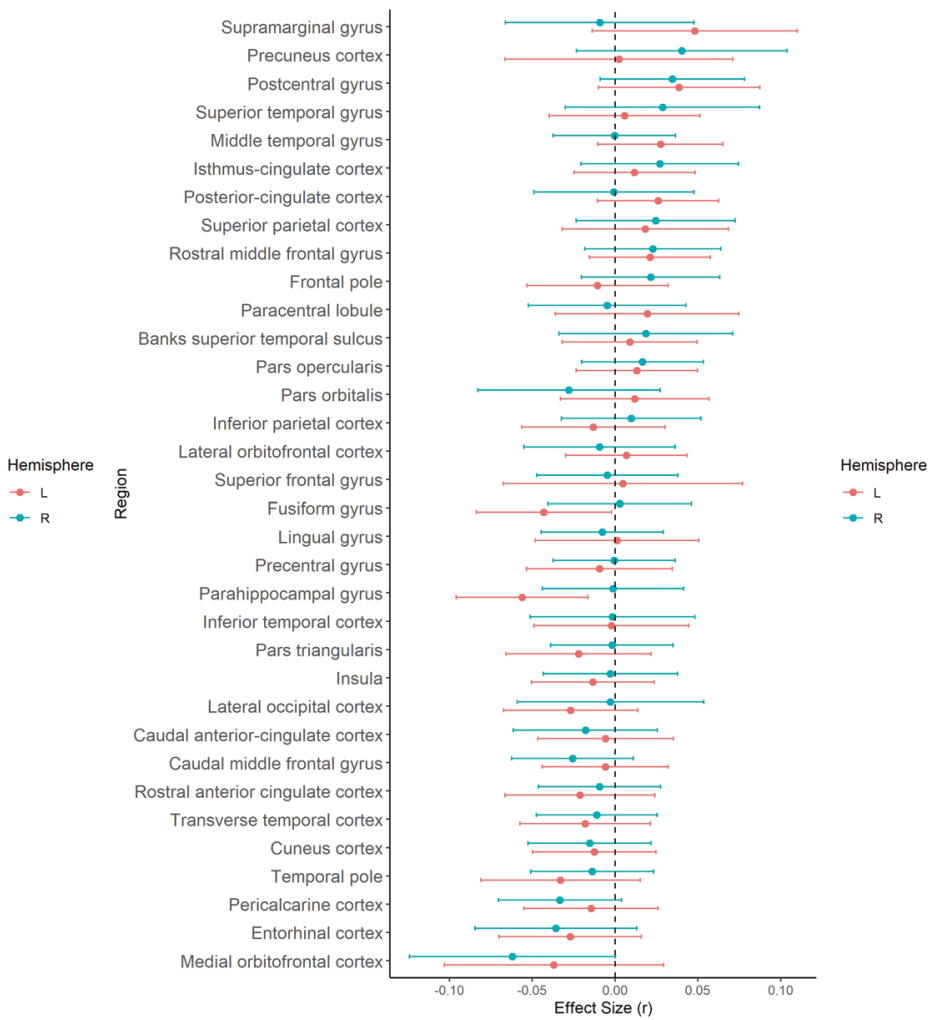

Fig. 1 Effect sizes of partial correlation ( $r$ ) between cortical thickness, surface area and schizotypy. A Cortical thickness, (B) Surface area. Effect sizes for all regions depicted were corrected for age, sex and global cortical thickness or total surface area, respectively. Red rectangle highlights effects surviving false discovery rate (FDR) correction $\left(p_{\mathrm{FDR}}<0.05\right)$.

A| Cortical thickness

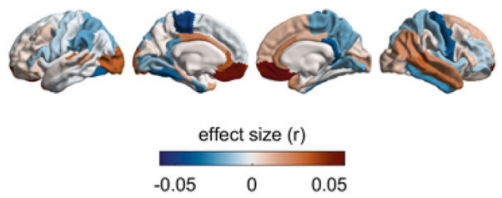

B| Surface area

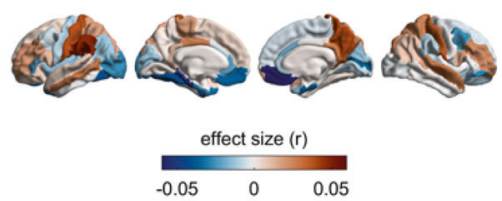

C| Forest plot of significant associations between mOFC/vmPFC thickness and schizotypy

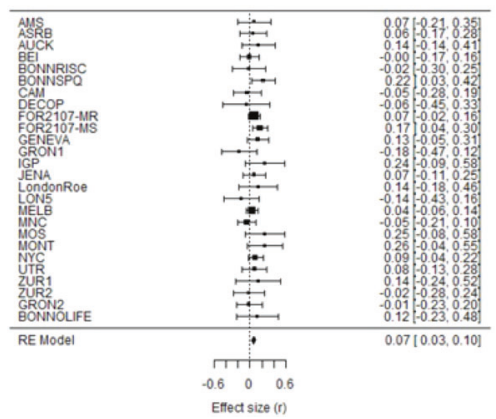

Fig. 2 Cortical maps of regional effect sizes for associations with schizotypy. A Cortical thickness, (B) Surface area. C Forest plot of the significant association between mOFC/vmPFC thickness and schizotypy, after false discovery rate (FDR) correction $\left(p_{\mathrm{FDR}}<0.05\right)$.

\section{DISCUSSION}

Leveraging neuroimaging data from 29 international sites, the present work is the first large-scale meta-analysis of cortical and subcortical measures in schizotypy, including over 3000 individuals. Our main finding was that greater CT in the mOFC/vmPFC was positively associated with higher schizotypy. Subcortical volume and SA analyses showed only subtle, non-significant correlations with schizotypy scores. These findings were, due to our healthy sample, free of potential influences of illness chronicity or antipsychotic medication on neuroanatomy. Moreover, we found a positive association between the schizotypy-related $C T$ pattern and the pattern of CT abnormalities observed in SZ, but not with that of $\mathrm{BD}$ or MDD, supporting the notion of neurobiological continuity across the extended psychosis phenotype. In contrast, patterns of 
schizotypy-related subcortical effects showed an unexpected negative association with patterns of subcortical volume abnormalities more generally, across the three psychiatric disorders.

The directionality of the observed association for $\mathrm{CT}$, reflecting thicker mOFC/vmPFC with higher schizotypy, is inverse to findings of prefrontal cortical thinning commonly observed in medicated patients with SZ [28, 47] and first-episode psychosis [27, 43]. However, our findings are in line with reports of greater OFC thickness in drug-naïve first-episode SZ patients [76, 77]. Prior schizotypy studies reported mixed findings, including greater prefrontal [38] or spared OFC thickness [36], and lower frontal gray matter volume $[37,42,46]$. Findings from studies in individuals at CHR for psychosis have likewise been inconsistent, with evidence for thicker OFC [78, 79], no differences [80] or thinner cortex [81] compared to healthy controls. This heterogeneity among these single studies may be partly explained by the use of different methods or limited power. On the other hand, there is growing

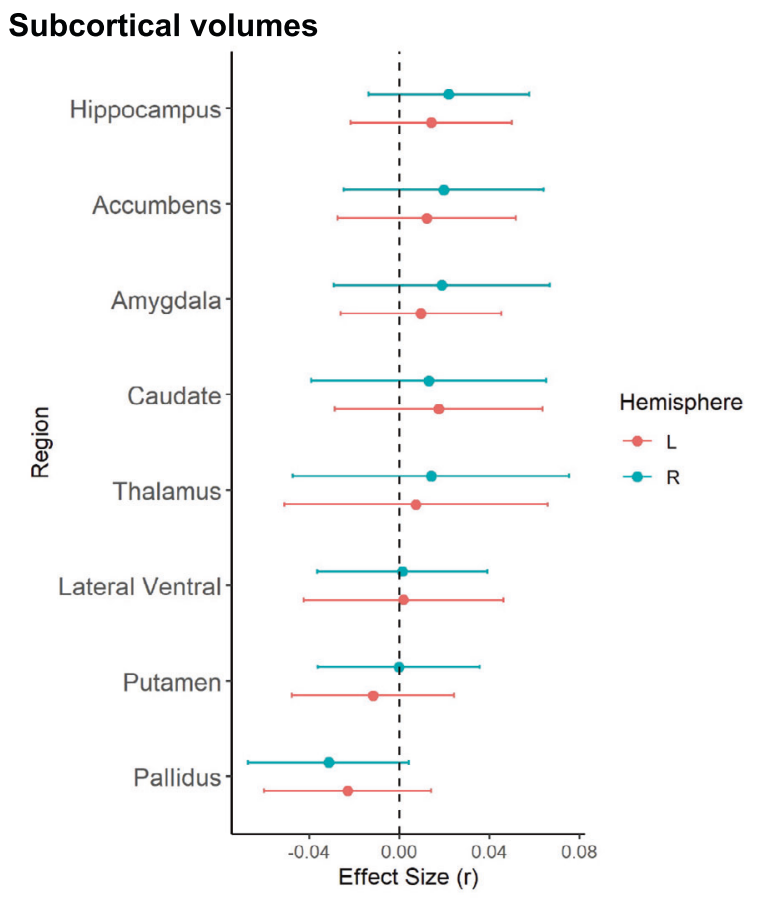

Fig. 3 Effect sizes of partial correlation ( $r$ ) between subcortical volumes and schizotypy. Effect sizes for all subcortical volumes depicted were corrected for age, sex and intracranial volume (ICV). evidence that variations of prefrontal CT are related to different atrisk phenotypes and the onset of early and late neurodevelopmental disturbances [25, 82]. Different anatomical trajectories have been reported in schizotypy [35], between different at-risk states [83] and particularly in CHR individuals who subsequently develop psychosis [82, 84]. Transition to psychosis has been associated with a steeper cortical thinning of heteromodal cortices, including the $\mathrm{mOFC} / \mathrm{vmPFC}$, compared to $\mathrm{CHR}$ individuals who do not convert to psychosis and healthy controls [85-88]. Thus, structural characteristics of the mOFC/vmPFC may vary based on the degree of risk and illness chronicity, from relatively thicker mOFC/vmPFC in healthy individuals with high schizotypy, to normal-to-elevated in CHR with lesser transition risk, to thinner in CHR individuals who convert to psychosis, to further thinning in chronic SZ.

The observed relationship between high schizotypy and greater mOFC/vmPFC thickness shares neuroanatomical similarities to other neurodevelopmental disorders such as autism spectrum disorders (ASD) (Cohen's $d=0.15, p_{\mathrm{FDR}}=0.0001$ [89]) and 22q deletion syndrome (Cohen's $d=0.61, p_{\mathrm{FDR}}<0.0001$ [69]). Such convergence aligns with observations of increased phenotypic expression of schizotypy in ASD [90, 91] and 22q deletion syndrome [92]. Together, these findings support the notion that high schizotypy may describe a predisposing trait of genetically and clinically overlapping phenotypes with SZ spectrum disorders and ASD [16, 93, 94]. Our cross-disorder whole-brain cortical mapping revealed a strong link between morphometric signatures of schizotypy with SZ, along a SZ-BD-MDD axis. These findings partly resemble previous genetic [95] and neuroanatomical correlations [70, 96] observed between SZ, BD and major depression [97]. Interestingly, the relationship between the cortical patterns of schizotypy and SZ revealed regional discordant and concordant effect sizes. Specifically, in schizotypy, the associations between higher schizotypy and lower CT were only observed in those regions showing the strongest negative effect sizes in SZ (e.g., thinning), such as the left and right fusiform gyrus or inferiotemporal gyrus. In other words, schizotypy and SZ showed somewhat concordant effect sizes in these regions. In contrast, discordant effect sizes of thinner CT in SZ and greater CT in high schizotypy were observed in other regions such as the rostral anterior cingulate, frontal pole and mOFC/vmPFC. Here, higher schizotypy was positively associated with greater $\mathrm{CT}$, while patients with SZ showed thinner cortex compared to controls (negative effect size). This neuroanatomical pattern of spatially distributed thinner and thicker cortex might reflect that in schizotypy samples, which comprise healthy individuals that will most likely not develop a full-blown psychotic disorder, effects may be subtle. Although speculative, the relative greater CT could a | Schizophrenia
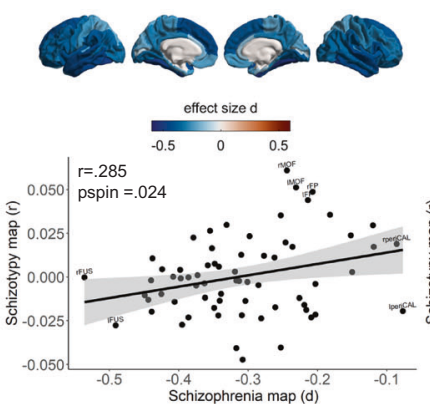

b | Bipolar Disorder
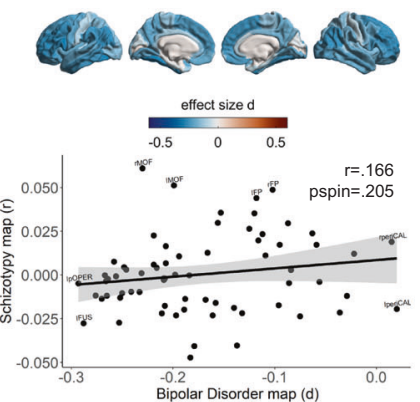

c | Major Depression
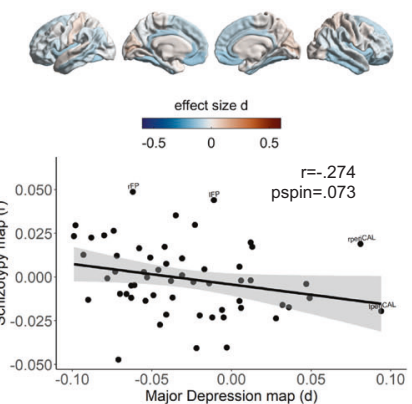

Fig. 4 Pattern similarity between cortical thickness effects in schizotypy and major psychiatric disorders. Abbreviations of the cortical regions are adopted from the brainGraph package and shown for regions with the most positive and negative effect sizes ( $r$ ): FUS fusiform gyrus, ITG inferior temporal gyrus, iCC isthmus cingulate cortex, MOF medial orbitofrontal cortex, paraC paracentral lobule, pOPER pars opercularis of inferior frontal gyrus, periCAL pericalcarine cortex, rACC rostral anterior cingulate cortex, FP frontal pole, INS insula. L left, $\mathrm{R}$ right. 
a | Schizophrenia

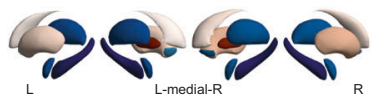

b | Bipolar Disorder

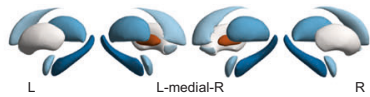

c | Major Depression

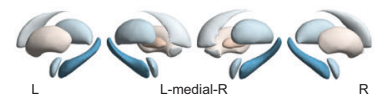

effect size d

\begin{tabular}{lll}
\multicolumn{2}{l}{ effect sized } \\
-0.2 & 0 & 0.2
\end{tabular}

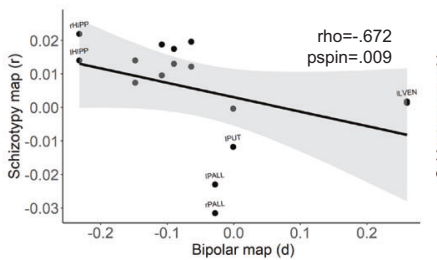

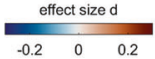

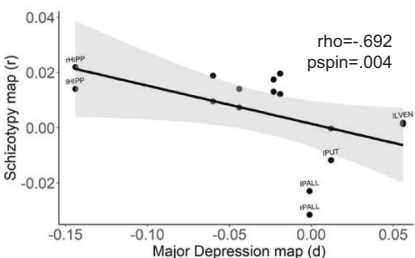

Fig. 5 Pattern similarity between subcortical volume effects in schizotypy and major psychiatric disorders. Abbreviations of subcortical regions are adopted from the brainGraph package and are shown for regions with the most positive and negative effect sizes ( $r$ ): HIPP hippocampus, PALL pallidum, PUT putamen, LVEN lateral ventricle, I left, $r$ right.

reflect either compensatory mechanisms or a pre-existing condition before the onset of accelerated thinning in those individuals that develop psychosis.

Whereas the cortex-wide similarity between schizotypy and SZ provides support for a specific relationship, schizotypy-related subcortical volumes showed a negative association with subcortical abnormalities in all three disorders (SZ, BD, MDD). Combined with the overall subtle links between schizotypy and subcortical volume, these inverse correlations may suggest that schizotypal traits are not directly linked to subcortical alterations of major psychiatric disorders in general, which may be better explained by other risk/disease factors (e.g., medication, disease course, common mental distress) [34, 66, 68], or that spared subcortical volume changes might be a protective factor in those individuals with high schizotypy and high resilience [16, 98, 99]. In line with the latter, first-degree relatives of BD patients showed higher than normal ICV (compared to controls), suggesting putative survivor effects in unaffected individuals with high liability for psychiatric disorders [100]. While an inverse subcortical pattern in schizotypy was not anticipated, further investigation and replication of these findings will be of interest.

Overall, our meta-analyses suggest that schizotypal traits in the general population are predominantly embedded in CT effects, while less associated with variations of subcortical volume or SA. The nature of preserved or greater cortical morphometry in schizotypy has been intensively debated, although the underlying cellular and molecular mechanisms remain unclear. Greater prefrontal CT might reflect abnormal or delayed cortical development [101-103], due to insufficient synaptic pruning [104] or altered cortical myelination $[105,106]$. Greater CT as a result of microstructural and cellular perturbations may therefore be associated with higher vulnerability, while SZ may only emerge in the presence of additional environmental, biological or genetic factors $[25,82,107]$. In line with this notion, several previous studies in CHR, genetic and clinical samples have shown that measures of schizotypy can be predictive for developing psychosis [1], although the predictive value of schizotypy might be sensitive to vulnerable periods (e.g., adolescence) and potentially increase with accumulation and interaction of other psychosis risk factors [26]. Alternatively, it has been proposed that neuroanatomical signatures such as preserved/greater prefrontal CT could reflect protective mechanisms for developing clinical symptoms of psychosis/SZ in high schizotypy individuals with absence of other risk factors $[16,99]$. This would align with a fully-dimensional model of schizotypy $[7,8]$, which predicts inverse associations in schizotypy samples consisting of healthy participants compared to samples of patients with SZ, supported by findings that frontal capacity is positively associated with schizotypy [99]. Large-scale longitudinal studies from neurodevelopment in childhood to early adulthood could help to further differentiate neuroanatomical trajectories of high schizotypy and their contribution to either psychosis in those individuals with concomitant risk factor [26] or to normative variations in otherwise healthy individuals [16].

\section{Limitations}

In interpreting the current findings, it is relevant to note that rather small effect sizes are typical for these types of large-scale neuroimaging meta-analysis and comparable effect size magnitudes have been reported in other ENIGMA studies of clinical populations $[67,89]$ and populations at higher risk for mental illnesses [100, 108]. Given that schizotypal traits were derived from healthy individuals in the general population, relationships between brain morphometry and schizotypy were expected to be even more subtle compared to clinical populations. The observed effect sizes can be positioned at the lower end of cortical effects in psychiatric disorders $(<\mathrm{SZ}$ and BD $[28,65])$ but are comparable to those reported for MDD and ASD $[67,89]$ ) as well as population neuroscience studies of associations between brain structure and polygenic risk for psychosis or psychotic symptoms [109, 110]. Furthermore, no moderating effects of the type of schizotypy questionnaire, FreeSurfer version, number of scanners, or scanner field strength were found supporting the strength of the observed findings. This first meta-analysis aimed at reflecting the large body of research on neuroanatomical patterns of total schizotypy within the psychosis continuum. However, the use of total schizotypy scores might have obscured some neuroanatomical differences related to distinct schizotypy dimensions (e.g., positive, negative and disorganized) [35, 111]. The ENIGMA Schizotypy Working Group is actively working on pooling data to map neuroanatomical patterns of separate schizotypy dimensions, which will be reported elsewhere [56]. In addition, while family history of psychiatric disorders was an exclusion criterion in many of the included cohorts [36, 37, 40, 41, 44, 45], these data were not available for all participants. Future prospective neuroimaging studies in schizotypy explicitly collecting information on participants' family history for psychiatric disorders would enable the investigation of potential moderating effects of family history on neuroanatomical pattern similarities between schizotypy and major psychiatric disorders. Finally, while we observed a specific relationship between the schizotypyrelated $\mathrm{CT}$ pattern and the pattern of case-control CT abnormalities previously found in SZ, these findings are derived by comparing effect size maps from large-scale patient populations and as such should not be interpreted as an equivalent to comparing individuals from different disease groups. To advance our understanding of the neurobiology of psychosis risk beyond MRI measurements associated with overall schizotypy levels, there will be continued effort by the ENIGMA Schizotypy Working Group 
to (i) include more datasets, (ii) incorporate genetic as well as multimodal neuroimaging data, and (iii) identify associations between MRI-derived measures and cell-type specific gene expression [97].

\section{CONCLUSION}

In summary, this is the first meta-analysis of neuroimaging data to comprehensively map the morphometric signature of schizotypy in healthy individuals. The results suggest a profile of CT abnormalities involving thicker prefrontal cortex related to more severe schizotypy. The CT pattern related to schizotypy was most closely linked to $\mathrm{CT}$ abnormalities in SZ, thus providing neuroanatomical support for dimensional continuity across the extended psychosis phenotype.

\section{REFERENCES}

1. Debbané M, Eliez S, Badoud D, Conus P, Flückiger R, Schultze-Lutter F. Developing psychosis and its risk states through the lens of schizotypy. Schizophr Bull. 2015;41:S396-407.

2. Everett KV, Linscott RJ. Dimensionality vs taxonicity of schizotypy: some new data and challenges ahead. Schizophr Bull 2015;41:S465-474.

3. van Os J, Linscott RJ, Myin-Germeys I, Delespaul P, Krabbendam L. A systematic review and meta-analysis of the psychosis continuum: evidence for a psychosis proneness-persistence-impairment model of psychotic disorder. Psychol Med. 2009;39:179-95.

4. Lenzenweger MF. Schizotaxia, schizotypy, and schizophrenia: Paul E. Meehl's blueprint for the experimental psychopathology and genetics of schizophrenia. J Abnorm Psychol. 2006;115:195-200.

5. Meehl PE. Schizotaxia, schizotypy, schizophrenia. Am Psychol. 1962;17:827-38.

6. Rado S. Dynamics and classification of disordered behavior. Am J Psychiatry. 1953;110:406-16.

7. Grant P, Green MJ, Mason OJ. Models of schizotypy: the importance of conceptual clarity. Schizophr Bull. 2018;44:S556-S563.

8. Claridge G. Schizotypy: implications for illness and health. New York, NY, US: Oxford University Press; 1997.

9. Kwapil TR, Barrantes-Vidal N. Schizotypy: looking back and moving forward. Schizophr Bull. 2015;41:S366-S373.

10. Vollema MG, van den Bosch RJ. The multidimensionality of schizotypy. Schizophr Bull. 1995;21:19-31.

11. Oezgen $M$, Grant $P$. Odd and disorganized-Comparing the factor structure of the three major schizotypy inventories. Psychiatry Res. 2018;267:289-95.

12. Cicero DC, Jonas KG, Li K, Perlman G, Kotov R. Common taxonomy of traits and symptoms: linking schizophrenia symptoms, schizotypy, and normal personality. Schizophr Bull. 2019;45:1336-48.

13. Schultze-Lutter F, Nenadic I, Grant P. Psychosis and Schizophrenia-Spectrum Personality Disorders Require Early Detection on Different Symptom Dimensions. Front Psychiatry. 2019;10:476.

14. Kemp KC, Bathery AJ, Barrantes-Vidal N, Kwapil TR. Positive, Negative, and Disorganized Schizotypy Predict Differential Patterns of Interview-Rated Schizophrenia-Spectrum Symptoms and Impairment: Assessment. 2020. 19 January 2020. https://doi.org/10.1177/1073191119900008.

15. Flückiger R, Ruhrmann S, Debbané $M$, Michel C, Hubl D, Schimmelmann BG, et al. Psychosis-predictive value of self-reported schizotypy in a clinical high-risk sample. J Abnorm Psychol. 2016;125:923-32.

16. Barrantes-Vidal N, Grant P, Kwapil TR. The role of schizotypy in the study of the etiology of schizophrenia spectrum disorders. Schizophr Bull. 2015;41:S408-416.

17. Kirschner M, Haugg A, Manoliu A, Simon JJ, Huys QJM, Seifritz E, et al. Deficits in context-dependent adaptive coding in early psychosis and healthy individuals with schizotypal personality traits. Brain J Neurol. 2018;141:2806-19.

18. Kirschner M, Hager OM, Muff L, Bischof M, Hartmann-Riemer MN, Kluge A, et al. Ventral Striatal Dysfunction and Symptom Expression in Individuals With Schizotypal Personality Traits and Early Psychosis. Schizophr Bull. 2018;44:147-57.

19. Modinos G, Egerton A, McLaughlin A, McMullen K, Kumari V, Lythgoe DJ, et al. Neuroanatomical changes in people with high schizotypy: relationship to glutamate levels. Psychol Med. 2018;48:1880-89.

20. Nelson MT, Seal ML, Pantelis C, Phillips LJ. Evidence of a dimensional relationship between schizotypy and schizophrenia: a systematic review. Neurosci Biobehav Rev. 2013;37:317-27.

21. Ronald A, Pain O. A systematic review of genome-wide research on psychotic experiences and negative symptom traits: new revelations and implications for psychiatry. Hum Mol Genet. 2018;27:R136-R152.
22. Sabaroedin K, Tiego J, Parkes L, Sforazzini F, Finlay A, Johnson B, et al. Functional Connectivity of Corticostriatal Circuitry and Psychosis-like Experiences in the General Community. Biol Psychiatry. 2019;86:16-24.

23. Sahakyan L, Meller T, Evermann U, Schmitt S, Pfarr J-K, Sommer J, et al. Anterior vs Posterior Hippocampal Subfields in an Extended Psychosis Phenotype of Multidimensional Schizotypy in a Nonclinical Sample. Schizophr Bull. 2020;21:2020 https://doi.org/10.1093/schbul/sbaa099. July.

24. Rapoport JL, Giedd JN, Gogtay N. Neurodevelopmental model of schizophrenia: update 2012. Mol Psychiatry 2012;17:1228-38.

25. Insel TR. Rethinking schizophrenia. Nature. 2010;468:187-93.

26. Debbané $M$, Barrantes-Vidal N. Schizotypy from a developmental perspective. Schizophr Bull 2015;41:S386-395.

27. Wannan CMJ, Cropley VL, Chakravarty MM, Bousman C, Ganella EP, Bruggemann JM, et al. Evidence for Network-Based Cortical Thickness Reductions in Schizophrenia. Am J Psychiatry. 2019;176:552-63.

28. van Erp TGM, Walton E, Hibar DP, Schmaal L, Jiang W, Glahn DC, et al. Cortical Brain Abnormalities in 4474 Individuals With Schizophrenia and 5098 Control Subjects via the Enhancing Neuro Imaging Genetics Through Meta Analysis (ENIGMA) Consortium. Biol Psychiatry. 2018;84:644-54.

29. Vieira S, Gong Q, Scarpazza C, Lui S, Huang X, Crespo-Facorro B, et al. Neuroanatomical abnormalities in first-episode psychosis across independent samples: a multi-centre mega-analysis. Psychol Med. 2021;51:340-50.

30. Fusar-Poli P, Borgwardt S, Crescini A, Deste G, Kempton MJ, Lawrie S, et al. Neuroanatomy of vulnerability to psychosis: a voxel-based meta-analysis. Neurosci Biobehav Rev. 2011;35:1175-85.

31. Koutsouleris N, Schmitt GJE, Gaser C, Bottlender R, Scheuerecker J, McGuire P, et al. Neuroanatomical correlates of different vulnerability states for psychosis and their clinical outcomes. Br J Psychiatry J Ment Sci. 2009;195:218-26.

32. Fervaha G, Remington G. Neuroimaging findings in schizotypal personality disorder: a systematic review. Prog Neuropsychopharmacol Biol Psychiatry. 2013:43:96-107.

33. van Lutterveld $R$, van den Heuvel MP, Diederen KMJ, de Weijer AD, Begemann $\mathrm{MJH}$, Brouwer RM, et al. Cortical thickness in individuals with non-clinical and clinical psychotic symptoms. Brain. 2014;137:2664-9.

34. van Erp TGM, Hibar DP, Rasmussen JM, Glahn DC, Pearlson GD, Andreassen OA, et al. Subcortical brain volume abnormalities in 2028 individuals with schizophrenia and 2540 healthy controls via the ENIGMA consortium. Mol Psychiatry. 2016;21:547-53.

35. Derome M, Tonini E, Zöller D, Schaer M, Eliez S, Debbané M. Developmental Trajectories of Cortical Thickness in Relation to Schizotypy During Adolescence. Schizophr Bull. 2020;5:2020 https://doi.org/10.1093/schbul/sbaa020. March.

36. DeRosse P, Nitzburg GC, Ikuta T, Peters BD, Malhotra AK, Szeszko PR. Evidence from structural and diffusion tensor imaging for frontotemporal deficits in psychometric schizotypy. Schizophr Bull. 2015;41:104-14.

37. Ettinger U, Williams SCR, Meisenzahl EM, Möller H-J, Kumari V, Koutsouleris N. Association between brain structure and psychometric schizotypy in healthy individuals. World J Biol Psychiatry Off J World Fed Soc. Biol Psychiatry. 2012;13:544-9.

38. Kühn S, Gallinat J. Quantitative meta-analysis on state and trait aspects of auditory verbal hallucinations in schizophrenia. Schizophr Bull. 2012;38:779-86.

39. Meller T, Schmitt $S$, Ettinger $U$, Grant $P$, Stein F, Brosch $K_{\text {, et al. Brain structural }}$ correlates of schizotypal signs and subclinical schizophrenia nuclear symptoms in healthy individuals. Psychol Med. 2020:1-10. https://doi.org/10.1017/ S0033291720002044. Epub ahead of print.

40. Modinos G, Mechelli A, Ormel J, Groenewold NA, Aleman A, McGuire PK. Schizotypy and brain structure: a voxel-based morphometry study. Psychol Med. 2010:40:1423-31.

41. Nenadic I, Lorenz C, Langbein K, Dietzek M, Smesny S, Schönfeld N, et al. Brain structural correlates of schizotypy and psychosis proneness in a non-clinical healthy volunteer sample. Schizophr Res. 2015;168:37-43.

42. Wiebels K, Waldie KE, Roberts RP, Park HRP. Identifying grey matter changes in schizotypy using partial least squares correlation. Cortex J Devoted Study Nerv Syst Behav. 2016;81:137-50.

43. Kirschner M, Schmidt A, Hodzic-Santor B, Burrer A, Manoliu A, Zeighami Y, et al. Orbitofrontal-Striatal Structural Alterations Linked to Negative Symptoms at Different Stages of the Schizophrenia Spectrum. Schizophr Bull. 2020;1:2020 https://doi.org/10.1093/schbul/sbaa169. December.

44. Modinos G, Egerton A, McLaughlin A, McMullen K, Kumari V, Lythgoe DJ, et al. Neuroanatomical changes in people with high schizotypy: relationship to glutamate levels. Psychol Med. 2018;48:1880-9.

45. Wang Y, Yan C, Yin D, Fan M, Cheung EFC, Pantelis C, et al. Neurobiological Changes of Schizotypy: Evidence From Both Volume-Based Morphometric Analysis and Resting-State Functional Connectivity. Schizophr Bull. 2015;41: S444-S454. 
46. Pfarr J-K, Nenadić I. A multimodal imaging study of brain structural correlates of schizotypy dimensions using the MSS. Psychiatry Res Neuroimaging. 2020;302:111104.

47. Walton E, Hibar DP, van Erp TGM, Potkin SG, Roiz-Santiañez R, Crespo-Facorro B, et al. Prefrontal cortical thinning links to negative symptoms in schizophrenia via the ENIGMA consortium. Psychol Med. 2018:48:82-94.

48. Walton E, Hibar DP, van Erp TG, Potkin SG, Roiz-Santiañez R, Crespo-Facorro B, et al. Positive symptoms associate with cortical thinning in the superior temporal gyrus via the ENIGMA-Schizophrenia consortium. Acta Psychiatr Scand. 2017;135:439-47.

49. Chapman LJ, Chapman JP, Raulin ML. Body-image aberration in schizophrenia. J Abnorm Psychol. 1978;87:399-407.

50. Chapman LJ, Chapman JP, Raulin ML. Scales for physical and social anhedonia. J Abnorm Psychol. 1976;85:374-82.

51. Eckblad M, Chapman LJ. Magical ideation as an indicator of schizotypy. J Consult Clin Psychol. 1983;51:215-25.

52. Stefanis NC, Hanssen M, Smirnis NK, Avramopoulos DA, Evdokimidis IK, Stefanis $\mathrm{CN}$, et al. Evidence that three dimensions of psychosis have a distribution in the general population. Psychol Med. 2002;32:347-58.

53. Raine A. The SPQ: a scale for the assessment of schizotypal personality based on DSM-III-R criteria. Schizophr Bull. 1991;17:555-64.

54. Mason O, Claridge G, Jackson M. New scales for the assessment of schizotypy. Personal Individ Differ. 1995;18:7-13.

55. Rust J. The Rust Inventory of Schizoid Cognitions (RISC): a psychometric measure of psychoticism in the normal population. Br J Clin Psychol. 1987;26:151-2.

56. Kirschner M, Hodzic-Santor B, Kircher T, Nenadic I, Fornito A, Green M, et al. T162. Thicker prefrontal cortex is associated with subclinical negative symptoms in schizotypy - an enigma consortium meta-analysis. Schizophr Bull. 2020;46: S292-S293.

57. Fischl B. FreeSurfer. Neurolmage 2012;62:774-81.

58. Fischl B, Salat DH, Busa E, Albert M, Dieterich M, Haselgrove $C$, et al. Whole Brain Segmentation: Automated Labeling of Neuroanatomical Structures in the Human Brain. Neuron. 2002;33:341-55.

59. Desikan RS, Ségonne F, Fischl B, Quinn BT, Dickerson BC, Blacker D, et al. An automated labeling system for subdividing the human cerebral cortex on MRI scans into gyral based regions of interest. Neurolmage. 2006;31:968-80.

60. Hanlon CA, Owens MM, Joseph JE, Zhu X, George MS, Brady KT, et al. Lower subcortical gray matter volume in both younger smokers and established smokers relative to non-smokers. Addict Biol. 2016;21:185-95.

61. Karama S, Ducharme S, Corley J, Chouinard-Decorte F, Starr JM, Wardlaw JM, et al. Cigarette smoking and thinning of the brain's cortex. Mol Psychiatry. 2015;20:778-85.

62. Viechtbauer W. Conducting Meta-Analyses in R with the metafor Package. J Stat Softw. 2010;36:1-48.

63. Benjamini $Y$, Hochberg Y. Controlling the false discovery rate: a practical and powerful approach to multiple testing. J R Stat Soc Ser B Methodol. 1995;57:289-300.

64. Storey JD. A Direct Approach to False Discovery Rates. J R Stat Soc Ser B Stat Methodol. 2002;64:479-98.

65. Hibar DP, Westlye LT, Doan NT, Jahanshad N, Cheung JW, Ching CRK, et al. Cortical abnormalities in bipolar disorder: an MRI analysis of 6503 individuals from the ENIGMA Bipolar Disorder Working Group. Mol Psychiatry. 2018;23:932-42.

66. Hibar DP, Westlye LT, van Erp TGM, Rasmussen J, Leonardo CD, Faskowitz J, et al. Subcortical volumetric abnormalities in bipolar disorder. Mol Psychiatry. 2016;21:1710-16.

67. Schmaal L, Hibar DP, Sämann PG, Hall GB, Baune BT, Jahanshad N, et al. Cortical abnormalities in adults and adolescents with major depression based on brain scans from 20 cohorts worldwide in the ENIGMA Major Depressive Disorder Working Group. Mol Psychiatry. 2017;22:900-9.

68. Schmaal L, Veltman DJ, van Erp TGM, Sämann PG, Frodl T, Jahanshad N, et al. Subcortical brain alterations in major depressive disorder: findings from the ENIGMA Major Depressive Disorder working group. Mol Psychiatry. 2016;21:806-12.

69. Sun D, Ching CRK, Lin A, Forsyth JK, Kushan L, Vajdi A, et al. Large-scale mapping of cortical alterations in 22q11.2 deletion syndrome: Convergence with idiopathic psychosis and effects of deletion size. Mol Psychiatry. 2020;25:1822-34. https://doi.org/10.1038/s41380-018-0078-5.

70. Opel N, Goltermann J, Hermesdorf M, Berger K, Baune BT, Dannlowski U. CrossDisorder Analysis of Brain Structural Abnormalities in Six Major Psychiatric Disorders: A Secondary Analysis of Mega- and Meta-analytical Findings From the ENIGMA Consortium. Biol Psychiatry. 2020;88:678-86. https://doi.org/10.1016/j. biopsych.2020.04.027.
71. Larivière S, Rodríguez-Cruces R, Royer J, Caligiuri ME, Gambardella A, Concha L, et al. Network-based atrophy modeling in the common epilepsies: a worldwide ENIGMA study. Sci Adv. 2020;6:eabc6457.

72. Alexander-Bloch AF, Shou H, Liu S, Satterthwaite TD, Glahn DC, Shinohara RT, et al. On testing for spatial correspondence between maps of human brain structure and function. Neurolmage. 2018;178:540-51.

73. Markello RD, Misic B. Comparing spatial null models for brain maps. NeuroImage. 2021;236:118052.

74. de Winter JCF, Gosling SD, Potter J. Comparing the Pearson and Spearman correlation coefficients across distributions and sample sizes: A tutorial using simulations and empirical data. Psychol Methods. 2016;21:273-90.

75. Diedenhofen B, Musch J. cocor: a Comprehensive Solution for the Statistical Comparison of Correlations. PLOS ONE. 2015;10:e0121945.

76. Ren W, Lui S, Deng W, Li F, Li M, Huang X, et al. Anatomical and functional brain abnormalities in drug-naive first-episode schizophrenia. Am J Psychiatry. 2013;170:1308-16.

77. Xiao Y, Lui S, Deng W, Yao L, Zhang W, Li S, et al. Altered Cortical Thickness Related to Clinical Severity But Not the Untreated Disease Duration in Schizophrenia. Schizophr Bull. 2015;41:201-10.

78. Dukart J, Smieskova R, Harrisberger F, Lenz C, Schmidt A, Walter A, et al. Agerelated brain structural alterations as an intermediate phenotype of psychosis. J Psychiatry Neurosci JPN. 2017;42:307-19.

79. Li X, Alapati V, Jackson C, Xia S, Bertisch HC, Branch CA, et al. Structural abnormalities in language circuits in genetic high-risk subjects and schizophrenia patients. Psychiatry Res Neuroimaging. 2012;201:182-9.

80. Klauser $\mathrm{P}$, Zhou J, Lim JKW, Poh JS, Zheng H, Tng HY, et al. Lack of Evidence for Regional Brain Volume or Cortical Thickness Abnormalities in Youths at Clinical High Risk for Psychosis: Findings From the Longitudinal Youth at Risk Study. Schizophr Bull. 2015;41:1285-93.

81. Jung $W H$, Kim JS, Jang JH, Choi J-S, Jung MH, Park J-Y, et al. Cortical thickness reduction in individuals at ultra-high-risk for psychosis. Schizophr Bull. 2011;37:839-49.

82. Chung Y, Addington J, Bearden CE, Cadenhead K, Cornblatt B, Mathalon DH, et al. Use of Machine Learning to Determine Deviance in Neuroanatomical Maturity Associated With Future Psychosis in Youths at Clinically High Risk. JAMA Psychiatry. 2018;75:960-8.

83. Buechler R, Wotruba D, Michels L, Theodoridou A, Metzler S, Walitza S, et al. Cortical Volume Differences in Subjects at Risk for Psychosis Are Driven by Surface Area. Schizophr Bull. 2020;28:2020 https://doi.org/10.1093/schbul/ sbaa066. May.

84. Cannon TD, Chung Y, He G, Sun D, Jacobson A, van Erp TGM, et al. Progressive Reduction in Cortical Thickness as Psychosis Develops: A Multisite Longitudinal Neuroimaging Study of Youth at Elevated Clinical Risk. Biol Psychiatry. 2015;77:147-57.

85. Borgwardt SJ, McGuire PK, Aston J, Gschwandtner U, Pflüger MO, Stieglitz R-D, et al. Reductions in frontal, temporal and parietal volume associated with the onset of psychosis. Schizophr Res. 2008;106:108-14.

86. Koutsouleris N, Kambeitz-llankovic L, Ruhrmann S, Rosen M, Ruef A, Dwyer DB, et al. Prediction Models of Functional Outcomes for Individuals in the Clinical High-Risk State for Psychosis or With Recent-Onset Depression: A Multimodal, Multisite Machine Learning Analysis. JAMA Psychiatry. 2018;75:1156-72.

87. Sun D, Phillips L, Velakoulis D, Yung A, McGorry PD, Wood SJ, et al. Progressive brain structural changes mapped as psychosis develops in 'at risk' individuals. Schizophr Res. 2009;108:85-92.

88. Takahashi T, Wood SJ, Yung AR, Phillips LJ, Soulsby B, McGorry PD, et al. Insular cortex gray matter changes in individuals at ultra-high-risk of developing psychosis. Schizophr Res. 2009;111:94-102.

89. van Rooij D, Anagnostou E, Arango C, Auzias G, Behrmann M, Busatto GF, et al. Cortical and Subcortical Brain Morphometry Differences Between Patients With Autism Spectrum Disorder and Healthy Individuals Across the Lifespan: Results From the ENIGMA ASD Working Group. Am J Psychiatry. 2018;175:359-69.

90. Gong J-B, Wang Y, Lui SSY, Cheung EFC, Chan RCK. Childhood trauma is not a confounder of the overlap between autistic and schizotypal traits: A study in a non-clinical adult sample. Psychiatry Res. 2017;257:111-7.

91. Zhou H-Y, Yang H-X, Gong J-B, Cheung EFC, Gooding DC, Park S, et al. Revisiting the overlap between autistic and schizotypal traits in the non-clinical population using meta-analysis and network analysis. Schizophr Res. 2019;212:6-14.

92. Fonseca-Pedrero $E$, Debbané $M$, Schneider $M$, Badoud D, Eliez S. Schizotypal traits in adolescents with 22q11.2 deletion syndrome: validity, reliability and risk for psychosis. Psychol Med. 2016;46:1005-13.

93. Guan J, Cai JJ, Ji G, Sham PC. Commonality in dysregulated expression of gene sets in cortical brains of individuals with autism, schizophrenia, and bipolar disorder. Transl Psychiatry. 2019;9:1-15. 
94. Lee SH, Ripke S, Neale BM, Faraone SV, Purcell SM, Perlis RH, et al. Genetic relationship between five psychiatric disorders estimated from genome-wide SNPs. Nat Genet. 2013;45:984-94.

95. Bulik-Sullivan B, Finucane HK, Anttila V, ReproGen Consortium, Psychiatric Genomics Consortium, Genetic Consortium for Anorexia Nervosa of the Wellcome Trust Case Control Consortium 3. et al. An atlas of genetic correlations across human diseases and traits. Nat Genet. 2015;47:1236-41.

96. NV Radonjić, JL Hess, P Rovira, O Andreassen, JK Buitelaar, CRK Ching, et al. Structural Brain Imaging Studies Offer Clues about the Effects of the Shared Genetic Etiology among Neuropsychiatric Disorders. bioRxiv809582; https://doi. org/10.1101/809582

97. Disorder WC for the A-D, Disorder AS, Disorder B, Disorder MD, Disorder O-C, Groups and SEW. et al. Virtual Histology of Cortical Thickness and Shared Neurobiology in 6 Psychiatric Disorders. JAMA Psychiatry. 2020;26:2020 https:// doi.org/10.1001/jamapsychiatry.2020.2694. August.

98. Rosell DR, Futterman SE, McMaster A, Siever LJ. Schizotypal personality disorder: a current review. Curr Psychiatry Rep. 2014;16:452.

99. Siever LJ, Davis KL. The pathophysiology of schizophrenia disorders: perspectives from the spectrum. Am J Psychiatry. 2004;161:398-413.

100. de Zwarte SMC, Brouwer RM, Agartz I, Alda M, Aleman A, Alpert Kl, et al. The Association Between Familial Risk and Brain Abnormalities Is Disease Specific: An ENIGMA-Relatives Study of Schizophrenia and Bipolar Disorder. Biol Psychiatry. 2019;86:545-56.

101. Tamnes CK, Herting MM, Goddings A-L, Meuwese R, Blakemore S-J, Dahl RE, et al. Development of the Cerebral Cortex across Adolescence: A Multisample Study of Inter-Related Longitudinal Changes in Cortical Volume, Surface Area, and Thickness. J Neurosci Off J Soc Neurosci. 2017;37:3402-12.

102. Gogtay N, Giedd JN, Lusk L, Hayashi KM, Greenstein D, Vaituzis AC, et al. Dynamic mapping of human cortical development during childhood through early adulthood. Proc Natl Acad Sci. 2004;101:8174-9.

103. Sowell ER, Thompson PM, Leonard CM, Welcome SE, Kan E, Toga AW. Longitudinal Mapping of Cortical Thickness and Brain Growth in Normal Children. J Neurosci. 2004;24:8223-31.

104. Rakic P. Specification of cerebral cortical areas. Science. 1988;241:170-6.

105. Romero-Garcia R, Seidlitz J, Whitaker KJ, Morgan SE, Fonagy P, Dolan RJ, et al. Schizotypy-Related Magnetization of Cortex in Healthy Adolescence Is Colocated With Expression of Schizophrenia-Related Genes. Biol Psychiatry. 2019;13:2019 https://doi.org/10.1016/j.biopsych.2019.12.005. December.

106. Whitaker KJ, Vértes $P E$, Romero-Garcia R, Váša $F$, Moutoussis $M$, Prabhu $G$, et al. Adolescence is associated with genomically patterned consolidation of the hubs of the human brain connectome. Proc Natl Acad Sci. 2016;113:9105-10.

107. Smieskova R, Fusar-Poli $P$, Allen $P$, Bendfeldt K, Stieglitz RD, Drewe J, et al. Neuroimaging predictors of transition to psychosis-a systematic review and meta-analysis. Neurosci Biobehav Rev. 2010;34:1207-22.

108. ENIGMA Clinical High Risk for Psychosis Working Group, Jalbrzikowski M, Hayes RA, Wood SJ, Nordholm D, Zhou JH, et al. Association of Structural Magnetic Resonance Imaging Measures With Psychosis Onset in Individuals at Clinical High Risk for Developing Psychosis: An ENIGMA Working Group Mega-analysis. JAMA Psychiatry. 2021;5:2021 https://doi.org/10.1001/jamapsychiatry.2021.0638. May.

109. Neilson E, Shen X, Cox SR, Clarke T-K, Wigmore EM, Gibson J, et al. Impact of Polygenic Risk for Schizophrenia on Cortical Structure in UK Biobank. Biol Psychiatry. 2019;22:2019 https://doi.org/10.1016/j.biopsych.2019.04.013. April.

110. Ward J, Lyall LM, Bethlehem RAI, Ferguson A, Strawbridge RJ, Lyall DM, et al. Novel genome-wide associations for anhedonia, genetic correlation with psychiatric disorders, and polygenic association with brain structure. Transl Psychiatry. 2019;9:1-9.

111. Evermann U, Gaser C, Besteher B, Langbein K, Nenadić I. Cortical Gyrification, Psychotic-Like Experiences, and Cognitive Performance in Nonclinical Subjects. Schizophr Bull. https://doi.org/10.1093/schbul/sbaa068.

\section{ACKNOWLEDGEMENTS}

Core funding for ENIGMA was provided by the NIH Big Data to Knowledge (BD2K) program under consortium grant U54 EB020403 (PI: PMT). This research was funded in whole, or in part, by the Wellcome Trust [Sir Henry Dale Fellowship 202397/Z/16/Z to GM]. For the purpose of open access, the author has applied a CC BY public copyright licence to any Author Accepted Manuscript version arising from this submission. MK acknowledges funding from the Swiss National Science Foundation
(P2SKP3_178175). Acknowledgments and funding details for the various participating data contributors are listed in at the end of the Supplementary Material.

\section{AUTHOR CONTRIBUTIONS}

Author contributions: MK, BH-S and GM had full access to all of the data in the study and take responsibility for the integrity of the data and the accuracy of the data analysis. MK and BH-S contributed equally to this work. Study concept and design: MK, TGMvanE, JAT, PMT, AA and GM. Acquisition, analysis, interpretation of data, and funding (in alphabetical order): $\mathrm{AA}, \mathrm{PA}, \mathrm{MA}, \mathrm{AAr}, \mathrm{BTB}, \mathrm{MAB}, \mathrm{BB}, \mathrm{BBes}, \mathrm{JB}, \mathrm{RC}, \mathrm{AD}, \mathrm{UD}$, MD, MDe, PDeR, KD, VE, UE, AKF, PF, AF, CG, JG, PG, MG, DG, MGru, CH, BH-S, SK, TK, MK, MKI, KK, SS-K, PK, AK, HK, VK, SL, IL, EJL, IL-J, J-BM, DM, TM, GM, AM, AMu, IN, CP, CPa, HRP-P, PP, YQ, WR, IS, LS, TJS, JT, PMT, AT, JAT, TGM-vanE, YW, KW. Statistical analysis: MK, BH-S, MA, J-BM. Drafting of the manuscript: MK, BH-S and GM. Critical revision of the manuscript for important intellectual content (in alphabetical order): $A A$, $\mathrm{PA}, \mathrm{MA}$, AAr, BTB, MAB, BB, BBes, JB, RC, AD, UD, MD, MDer, PDeR, KD, VE, UE, AKF, PF, AFor, CG, JGi, PG, MGr, DG, MGr, CH, BH-S, SK, TK, MK, MKI, KK, SS-K, PK, AK, HK, VK, SL, IL, EJL, IL-J, J-BM, DM, TM, GM, AM, AMu, IN, CP, CPaq, HRP-P, PPr, YQ, WR, IS, LS, TJS, JT, PM-Th, AT, JA-T, TGM-vanE, YW, KW. All authors approved the contents of the paper.

\section{FUNDING}

The funders had no role in the design and conduct of the study; collection, management, analysis, and interpretation of the data; preparation, review, or approval of the paper; and decision to submit the paper for publication.

\section{COMPETING INTERESTS}

PMT received partial grant support from Biogen, Inc. (Boston, USA) for work unrelated to this paper. SK received speaker honoraria from Janssen, Takeda, Lundbeck and Roche. Royalties for cognitive test and training software from Schuhfried. In the past 3 years, CP served on an advisory board for Lundbeck, Australia Pty Ltd. He has received honoraria for talks presented at educational meetings organized by Lundbeck. No other disclosures were reported.

\section{ADDITIONAL INFORMATION}

Supplementary information The online version contains supplementary material available at https://doi.org/10.1038/s41380-021-01359-9.

Correspondence and requests for materials should be addressed to Gemma Modinos.

Reprints and permission information is available at http://www.nature.com/ reprints

Publisher's note Springer Nature remains neutral with regard to jurisdictional claims in published maps and institutional affiliations.

\section{(i)}

Open Access This article is licensed under a Creative Commons Attribution 4.0 International License, which permits use, sharing, adaptation, distribution and reproduction in any medium or format, as long as you give appropriate credit to the original author(s) and the source, provide a link to the Creative Commons license, and indicate if changes were made. The images or other third party material in this article are included in the article's Creative Commons license, unless indicated otherwise in a credit line to the material. If material is not included in the article's Creative Commons license and your intended use is not permitted by statutory regulation or exceeds the permitted use, you will need to obtain permission directly from the copyright holder. To view a copy of this license, visit http://creativecommons. org/licenses/by/4.0/.

(c) The Author(s) 2021

${ }^{1}$ McConnell Brain Imaging Centre, Montréal Neurological Institute, McGill University, Montréal, QC, Canada. ${ }^{2}$ Department of Psychiatry, Psychotherapy and Psychosomatics, Psychiatric Hospital, University of Zurich, Zurich, Switzerland. ${ }^{3}$ Department of Psychosis Studies, King's College London, London, UK. ${ }^{4}$ University of Marburg, Marburg, Germany. ${ }^{5}$ Department of Psychiatry and Psychotherapy, University of Bonn, Bonn, Germany. ${ }^{6}$ Department of Psychiatry, University of Münster, Münster, Germany. ${ }^{7}$ Turner

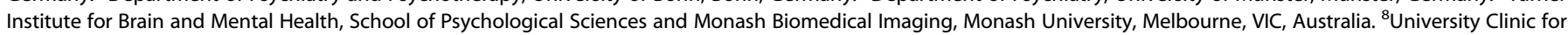


Radiology, University of Münster, Münster, Germany. ${ }^{9}$ Division of Psychiatry Research, Zucker Hillside Hospital, Northwell Health, Glen Oaks, NY, USA. ${ }^{10}$ The Feinstein Institutes for Medical Research, Center for Psychiatric Neuroscience, Manhasset, NY, USA. ${ }^{11}$ Department of Psychiatry, Donald and Barbara Zucker School of Medicine at Hofstra/Northwell, Hempstead, NY, USA. ${ }^{12}$ Department of Psychiatry, Melbourne Medical School, University of Melbourne, Melbourne, VIC, Australia. ${ }^{13}$ School of Psychiatry, University of New South Wales (UNSW), Sydney, NSW, Australia. ${ }^{14}$ Neuroscience Research Australia (NeuRA), Randwick, NSW, Australia. ${ }^{15}$ Melbourne Neuropsychiatry Centre, University of Melbourne, Melbourne, VIC, Australia. ${ }^{16}$ Institute of Psychology, Chinese Academy of Sciences, Beijing, China. ${ }^{17}$ University of Bonn, Bonn, Germany. ${ }^{18}$ University of Geneva, Geneva, Switzerland. ${ }^{19}$ Jena University Hospital, Jena, Germany. ${ }^{20}$ Department of Psychiatry, University of Cambridge, Cambridge, UK. ${ }^{21}$ Psychiatric University Hospital Zurich, University of Zurich, Zurich, Switzerland. ${ }^{22}$ Department of Psychiatry and Psychotherapy, Charité University Medicine, Berlin, Germany. ${ }^{23}$ Institute of Psychiatry, School of Medicine, University of São Paulo, São Paulo, Brazil. ${ }^{24}$ Brunel University London, Uxbridge, UK. ${ }^{25}$ School of Psychology, University of Auckland, Auckland, New Zealand. ${ }^{26}$ Free University, Amsterdam, the Netherlands. ${ }^{27}$ University of Roehampton, London, UK. ${ }^{28}$ Department of Biomedical Sciences of Cells and Systems, University Medical Center Groningen, University of Groningen, Groningen, the Netherlands. ${ }^{29}$ Mental Health Research Center, Moscow, Russian Federation. ${ }^{30}$ Department of Psychiatry, Geneva University Hospital, Geneva, Switzerland. ${ }^{31}$ City, University London, London, UK. ${ }^{32}$ Fresenius University of Applied Sciences, Frankfurt am Main, Germany. ${ }^{33}$ Clinical Translational Neuroscience Laboratory, Department of Psychiatry and Human Behavior, University of California Irvine, Irvine, CA, USA. ${ }^{34}$ Center for the Neurobiology of Learning and Memory, University of California Irvine, Irvine, CA, USA. ${ }^{35}$ Imaging Genetics and Neuroinformatics Lab, Georgia State University, Atlanta, GA, USA. ${ }^{36}$ Imaging Genetics Center, Mark and Mary Stevens Neuroimaging \& Informatics Institute, Keck School of Medicine of the University of Southern California, Marina del Rey, CA, USA. ${ }^{37}$ MRC Centre for Neurodevelopmental Disorders, King's College London, London, UK. ${ }^{38}$ These authors contributed equally: Matthias Kirschner, Benazir Hodzic-Santor. ${ }^{凶}$ email: gemma.modinos@kcl.ac.uk 